\section{Norovirus infection: why are the genogroup II genotype 4 strains so persistent in the population?}
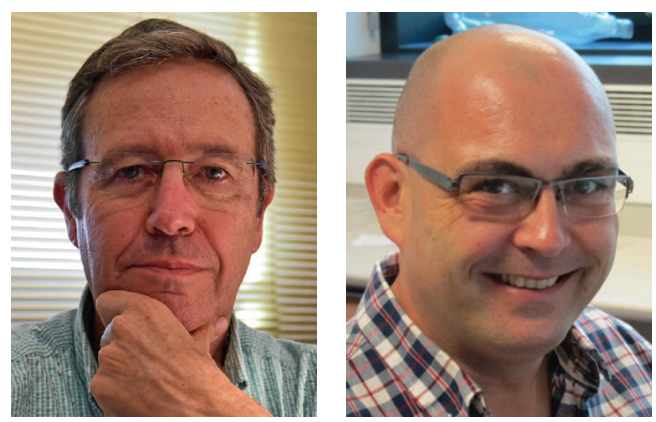

\author{
"The dynamics of genetic and \\ antigenic changes, a large \\ susceptible host population \\ and high viral fitness result in \\ a very efficient transmission \\ rate."
}

Javier Buesa ${ }^{*, 1,2}$ \& Jesús Rodríguez-Díaz ${ }^{1,2}$

First draft submitted: 28 September 2016; Accepted for publication: 7 October 2016; Published online: 9 November 2016

Human noroviruses (HuNoVs) are the predominant etiological agents of acute gastroenteritis worldwide, causing outbreaks and the majority of endemic illness across all age groups [1]. In many countries, $\mathrm{HuNoVs}$ have become the main cause of infantile gastroenteritis after the introduction of rotavirus vaccines and they have also been recognized globally as the main cause of associated foodborne diseases [2]. The improvements in the tools for the detection of $\mathrm{HuNoV}$ infection have also increased the number of cases reported for this disease, and it has been discussed whether this increase has been caused by a higher awareness or reflects emergence of new genogroup II genotype 4 (GII.4) strains [3]. Although the disease is usually self-limiting, in the United States, norovirus $(\mathrm{NoV})$ gastroenteritis causes an estimated 56,000-71,000 hospitalizations and 570-800 deaths each year [4]. Strains of the GII.4 genotype caused $70-80 \%$ of all the reported outbreaks over the past decade, and this genotype is more often associated with person-to-person than with foodborne transmission of the infection [3].

\section{HuNoV diversity \& evolution}

NoVs are genetically diverse positivestranded RNA viruses that are divided into seven genogroups (GI-GVII). Most $\mathrm{HuNoVs}$ belong to genogroups GI and GII, which are further subdivided into nine and 22 genotypes, respectively [4]. NoVs, similar to other RNA viruses, exist as a dynamic, rapidly evolving and genetically diverse population, under the rules of the viral quasispecies. This high level of genetic diversity has been considered the basis for their adaptability. It is thought that high mutation rates allow NoVs to evolve fast and to undergo rapid immune escape at the population level [5].

The NoV GII.4 epidemiology resembles that of influenza A viruses, with new antigenic variants emerging every $2-3$ years that replace the previously established variant.

\section{KEYWORDS}

- capsid protein epitopes $\bullet$ evolution

- Gll.4 genotype $\bullet$ herd immunity

- histo-blood group antigens

- norovirus • seroprevalence

- viral fitness $\bullet$ viral gastroenteritis 
"...mutations within the P2 region of the norovirus genogroup || genotype 4 variants capsid may affect the structural

conformation of the virions within the blocking antibody epitopes and the HBGA-binding sites, driving the evolution of genogroup I| genotype 4 variants."
Despite having a very high genetic diversity, most $\mathrm{HuNoV}$ infections, both as sporadic cases and as institutional outbreaks of acute gastro-enteritis, are caused by GII.4 genotype strains. In the last two decades, GII.4 genotype has caused six pandemics of acute gastroenteritis $[3,6]$. These pandemics spread rapidly across the globe, causing great economic burden due to medical and social expenses. Genotype GII.4 is further divided into strains or variants $[6,7]$. The GII.4 genotype variants associated with the majority of NoV pandemics have been GII.4 US1995/1996, Farmington_Hills_2002, GII.4 Hunter_2004, GII.4 Den Haag_2006b, GII.4 New Orleans_2009 and GII.4 Sydney_2012. It has been postulated that the first four of these six pandemic strains are the result of the mutational evolution of the P domain capsid, whereas the two most recent variants display additional recombination events between open reading frame (ORF)-1 and ORF2 [8,9].

It was suggested that the GII.4 viruses undergo epochal evolution, with the emergence of a new GII.4 variant coincidental with dramatic increases in the numbers of cases of acute gastroenteritis. The epidemiological impact of GII.4 genotype has been attributed to a combination of factors, including rapid nucleotide substitutions and homologous recombination [8]. Recombination typically occurs at the ORF1/2 overlap, although they have been also reported within ORF2 and at the ORF2/3 junction [8]. The accumulation of mutations in the P domain, the most protruding part of the capsid protein, correlates with the occurrence of genetic drift and selection of viral strains. Although the new epidemic variants differed by up to 25 aminoacid mutations, it was observed that consistent changes occurred in only five positions [7]. A significant association between the genetic findings and changes in the epidemiological features of NoV infections suggests that herd immunity plays a role in the epochal evolution of GII.4 strains, acting as an evolutionary driving force [7].

In the winter of 2014-2015 a novel GII.17 $\mathrm{HuNoV}$ genotype emerged as a major cause of gastroenteritis outbreaks in China and Japan. These novel viruses have been detected in sporadic cases throughout the world and an alert has been issued that they could replace the currently dominant GII.4 viruses [10]. The near future will witness whether the GII.17 strain turns into a pandemic strain or not.

\section{GII.4 NoV antigenicity}

Antigenic changes are generated through variations in the protruding P2 domain of the viral capsid protein $[11,12]$. These changes mostly occur at five antigenic sites within the P2 domain, referred to as epitopes $\mathrm{A}-\mathrm{E}$, that have been shown to be blockade epitopes using a surrogate neutralization assay [13]. As the P2 domain also contains the histo-blood group antigen (HBGA) binding site, antigenic changes may correlate with changes in HBGA-binding specificity. The HBGA-binding properties of the genotype GII.4 variants have changed over time, contributing to the high prevalence of genotype GII.4 [14]. These variations in HBGA-binding preferences of different GII.4 strains may determine the susceptibility of the population, according to the expression of HBGAs by different individuals. HBGAs are glycans expressed on the surface of specific cells and present as soluble molecules in saliva and other body secretions. A broader binding profile to HBGAs and a higher evolution rate might explain why GII.4 NoVs now predominate over NoVs from other genotypes. However, it has been reported that the occurrence of new variants do not correlate exactly with new and expanded binding profiles and that changes in amino acids that are not directly involved in HBGA attachment might contribute to the creation or the establishment of new binding profiles of GII.4 variants [14,15].

Several studies have shown that the mutations within the P2 region of the NoV GII.4 variants capsid may affect the structural conformation of the virions within the blocking antibody epitopes and the HBGA-binding sites, driving the evolution of GII.4 variants. These structural changes have favored the emergence of antibody escape variants and have changed the HBGA-binding properties of GII.4 variants over the time. Two of the main epitopes of NoV GII.4 are called antigenic site A (amino acids 296-298) and antigenic site B (amino acids 393-395), also named epitope D. They were defined after evolutionary and structural approaches [11] and confirmed using chimeric proteins and monoclonal antibodies [16]. Antigenic sites A and B have evolved over time explaining how the new variants can escape from the antibody pressure. Another study showed that antigenic site $\mathrm{A}$ is also formed by amino acids 294, 368 and 372 [12]. In addition to antigenic sites $\mathrm{A}$ and $\mathrm{B}$, we defined, by phage display and site-directed mutagenesis, another antigenic 
region recognized by a GII.4 blocking monoclonal antibody, 3C3G3. This region included two residues within the P2 domain (amino acids 397 and 448). These residues have evolved over time in a similar way to antigenic regions $\mathrm{A}$ and B. Site-directed mutagenesis experiments showed that changes in these two residues are enough to escape from antibody recognition [17]. Interestingly, the antigenic site B overlaps with HBGA-binding site 2 (amino acids 387-396), and it has been shown that different variants recognize different $\mathrm{HBGAs}$ due to the changes in the antigenic site $B$ [12]. Furthermore, we also observed a change in HBGA recognition pattern after site-directed mutagenesis of only one residue, amino acid 397 [17].

\section{Seroprevalence to genotype GII.4}

Previous work by our group showed a very high seroprevalence of antibodies against genotype GII.4 in our population, as well as an early-age $\mathrm{NoV}$ GII.4-specific antibody acquisition [18]. In order to measure the impact of exposure to $\mathrm{HuNoVs}_{\mathrm{N}}$ in the first years of life in The Netherlands, sera of young children were selected from three crosssectional population-based serum cohorts collected in 1963, 1983 and 2006/2007, and tested with a multiplex protein array to detect antibody responses to individual $\mathrm{NoV}$ genotypes [19]. It was found that the seroprevalence of GII.4-specific antibodies has significantly increased over the last decades, with concomitant reduction in exposure to GI and GIV NoVs, suggesting that the high GII.4 genotype incidence in very young children is a recent phenomenon [19].

\section{Replication of GIl.4 in human enteroids}

After 40 years of failing to replicate $\mathrm{HuNoV}_{\mathrm{s}}$ in routine cell cultures, the successful in vitro cultivation of several $\mathrm{HuNoV}$ strains in stem cell derived human intestinal enteroids has been recently reported [20]. Interestingly, some $\mathrm{HuNoV}$ genotypes (GII.3, GII.17, GI.1) require the presence of bile from different sources to replicate, whereas the addition of bile is not required for the replication of GII.4 strains, although it does enhance virus production. These differences in strain-specific growth requirements may indicate a higher viral fitness of GII.4 strains to replicate in enterocytes and to produce greater virus yields than other genotypes. This can also result in a higher level of virus shedding and an efficient transmission to the environment and to susceptible individuals, enhancing the infectivity of GII.4 strains and its persistence in the population.

\section{Conclusion \& future perspective}

The GII.4 genotype has been the predominant $\mathrm{HuNoV}$ for more than two decades. The dynamics of genetic and antigenic changes, a large susceptible host population and high viral fitness result in a very efficient transmission rate. Surveillance of HuNoVs circulating in the world population will provide knowledge about the future evolution of genotypes and GII.4 variants. This information will be relevant for the design of policies to prevent $\mathrm{HuNoV}$ infections.

\section{Acknowledgements}

The authors are grateful to $N$ Carmona-Vicente, $S$ Vila-Vicent, R Gozalbo-Robira, C Santiso-Bellón and co-workers at the Department of Microbiology, School of Medicine, University of Valencia.

\section{Financial \& competing interests disclosure}

This work has been financed by a grant (SAF2012-38368) to J Buesa and a grant (AGL2014-52996-C2-2-R) to $J$ Rodríguez-Diaz from the Ministerio de Economía $y$ Competitividad (MINECO), Spain. The authors declare that they have no other relevant affiliations or financial involvement with any organization or entity with a financial interest in or financial conflict with the subject matter.

No writing assistance was utilized in the production of this manuscript.

\section{References}

1 Ahmed SM, Hall AJ, Robinson AE et al. Global prevalence of norovirus in cases of gastroenteritis: a systematic review and meta-analysis. Lancet Infect. Dis. 14(8), 725-730 (2014).

2 Kirk MD, Pires SM, Black RE et al. Correction: World Health Organization estimates of the global and regional disease burden of 22 foodborne bacterial, protozoal, and viral diseases, 2010: a data synthesis. PLoS Med. 12(12), e1001940 (2015).

3 de Graaf M, van Beek J, Koopmans MP. Human norovirus transmission and evolution in a changing world. Nat. Rev. Microbiol. 14(7), 421-433 (2016).

4 Vinje J. Advances in laboratory methods for detection and typing of norovirus. J. Clin. Microbiol. 53(2), 373-381 (2015).
5 Cuevas JM, Combe M, Torres-Puente M et al. Human norovirus hyper-mutation revealed by ultra-deep sequencing. Infect. Genet. Evol. 41, 233-239 (2016).

6 Kroneman A, Vega E, Vennema $\mathrm{H}$ et al. Proposal for a unified norovirus nomenclature and genotyping. Arch. Virol. 158(10), 2059-2068 (2013).

7 Siebenga JJ, Vennema H, Renckens B et al. Epochal evolution of GGII.4 norovirus capsid 
proteins from 1995 to 2006. J. Virol. 81(18), 9932-9941 (2007).

8 Eden JS, Tanaka MM, Boni MF, Rawlinson WD, White PA. Recombination within the pandemic norovirus GII.4 lineage. J. Virol. 87(11), 6270-6282 (2013).

9 White PA. Evolution of norovirus. Clin. Microbiol. Infect. 20 (8), 741-745 (2014).

10 de Graaf M, van Beek J, Vennema $\mathrm{H}$ et al. Emergence of a novel GII.17 norovirus end of the GII.4 era? Euro Surveill. 20(26), pii: 21178 (2015).

11 Allen DJ, Gray JJ, Gallimore CI, Xerry J, Iturriza-Gómara M. Analysis of amino acid variation in the P2 domain of the GII- 4 norovirus VP1 protein reveals putative variant-specific epitopes. PLoS ONE 3(1), e1485 (2008).

12 Debbink K, Donaldson EF, Lindesmith LC, Baric RS. Genetic mapping of a highly variable norovirus GII.4 blockade epitope: potential role in escape from human herd immunity. J. Virol. 86(2), 1214-1226 (2012).

13 Lindesmith LC, Beltramello M, Donaldson EF et al. Immunogenetic mechanisms driving norovirus GII.4 antigenic variation. PLoS Pathog. 8(5), e1002705 (2012).

14 de Rougemont A, Ruvoen-Clouet N, Simon B et al. Qualitative and quantitative analysis of the binding of GII.4 norovirus variants onto human blood group antigens. J. Virol. 85(9), 4057-4070 (2011).

15 Lindesmith LC, Debbink K, Swanstrom J et al. Monoclonal antibody-based antigenic mapping of norovirus GII.4 - 2002. J. Virol. 86(2), 873-883 (2012).

16 Allen DJ, Noad R, Samuel D, Gray JJ, Roy P, Iturriza-Gomara $\mathrm{M}$. Characterisation of a GII-4 norovirus variant-specific surfaceexposed site involved in antibody binding. Virol. J. 6, 150 (2009).
17 Carmona-Vicente N, Vila-Vicent S, Allen D et al. Characterization of a novel conformational GII.4 norovirus epitope: implications for norovirus-host interactions. J. Virol. 90 (17), 7703-7714 (2016).

18 Carmona-Vicente N, Fernandez-Jimenez M, Ribes JM et al. Norovirus infections and seroprevalence of genotype GII.4-specific antibodies in a Spanish population. J. Med. Virol. 87(4), 675-682 (2015).

19 van Beek J, de Graaf M, Xia M et al. Comparison of norovirus genogroup I, II, and IV seroprevalence among children in The Netherlands, 1963, 1983, and 2006. J. Gen. Virol. 97(9), 2255-2264 (2016).

20 Ettayebi K, Crawford SE, Murakami K et al. Replication of human noroviruses in stem cell-derived human enteroids. Science 353(6306), 1387-1393 (2016). 